\title{
Phenotype Diversity Analysis of Amomum tsao-ko in Lvchun County of Yunnan Province
}

\author{
Shaoze Duan, Kunlong Zhu, Wenqiang Li, Linyan Xie, Xianwang Zhou, \\ Dong Shen, Tiantao Wang, Shenxuan Yang, Mengli Ma ${ }^{a}$ and Bingyue $\mathrm{Lu}^{\mathrm{b}}$
}

College of Life Science and Technology/ Key Laboratory of Crop High Quality and Efficient Cultivation and Security Control of College in Yunnan Province, Honghe University, Yunnan Mengzi 661100, China

amamlsky@126.com, blby202@126.com

Corresponding author: Bingyue Lu

Keywords: Amomum tsao-ko; Phenotype diversity; PCA.

\begin{abstract}
Genetic diversity analysis is very important for germplasm conservation and utilization. In this study, 13 quantitative traits of 50 Amomum tsao-ko plants were assessed by basic statistical parameters and principal component analysis (PCA). The results indicated that the phenotypic diversity was abundant in Amomum tsao-ko. Coefficients of variation $(\mathrm{CV})$ ranged from $7.60 \%$ to $43.86 \%$, and the largest of variation coefficient was the fruiting rate, while fresh fruit width was low. Shannon-Weaver diversity index ( $\left.\mathrm{H}^{\prime}\right)$ of 13 traits ranged from 1.71 to 2.22 , the largest and the smallest H' values were observed in seed regiment weight and fresh fruit weight, respectively. The principal component analysis (PCA) explained $78.381 \%$ of the total variation in four components. The first principal component was determined by dry fruit weight, fresh fruit width, dry fruit peel weight and seed regiment weight. The second was determined by fresh fruit length, dry fruit length and ratio of dry fruit length and width. The third mainly represented number of seeds per fruit, and the fourth reflected fresh fruit weight. Increasing the first principal component factor will be favorable for increasing the fruit weight, while the second principal component of the change will affect the shape of fruit.
\end{abstract}

\section{Introduction}

Amomum tsao-ko is a kind of perennial evergreen fasciculate herbaceous plant under Amomum of Zingiberaceae and the whole plant has spicy smell. Mainly distributed in Yunnan, Guangxi, Guizhou, Vietnam and other regions, it is a type of economic crop growing in humid forests in tropical and subtropical areas. The fruit of Amomum tsao-ko is a commonly used traditional Chinese medicine with the effects of eliminating dampness and phlegm as well as warming spleen and dispelling cold, which can also be used as a condiment in cooking [1]. The Amomum tsao-ko is listed as "Near Threatened' because its edible fruits have been over-harvested for trading [2]. The study of genetic diversity is very important for the conservation and utilization of the Amomum tsao-ko resources, but few detailed studies having been reported on the genetic diversity of the Amomum tsao-ko germplasm. Phenotypic diversity is one of the main methods of genetic diversity research. Identification using morphological traits is quick, intuitive and easily distinguishable. It is much acceptable to do preliminarily identify for an unknown cultivar [3]. Yunnan province, the main producing areas of Amomum tsao-ko yield accounted for $90 \%$ of China, Lvchun County is one of the major producing areas of Yunnan Province. By the end of 2015, Lvchun County cultivation area reached 11,500 hectares. The present study was initiated to assess the phenotypic diversity in 50 Amomum tsao-ko germplasm in Lvchun County of Yunnan Province according thirteen phenotypic characteristics. This will provide a theoretical basis for the conservation and utilization of Amomum tsao-ko resources. 


\section{Materials and Methods}

\subsection{Plant material and measurements}

50 Amomum tsao-ko plants were used in this study, which collected from Lvchun County of Yunnan Province $\left(22^{\circ} 89^{\prime} \mathrm{N}\right.$ and $102^{\circ} 41^{\prime}$ E, 1880 m.a.s.1.) in 2015. For each plant, a sample of three ears was randomly selected to perform morphometric analysis. The study was based on 13 traits, number of flowers per ear, fruiting rate, fresh fruit weight, fresh fruit length, fresh fruit width, ratio of fresh fruit length and width, dry fruit length, dry fruit width, ratio of dry fruit length and width, dry fruit weight, dry fruit peel weight, seed regiment weight, number of seeds per fruit. Parameters related to the fruit were measured at harvest stage and after harvest. Measurements were performed in triplicate. All measurements were performed using digital vernier caliper, precision weighing balance and observation calculation.

\subsection{Data analysis}

Basic statistical parameters and principal component analysis (PCA) were determined for all characters evaluated, using the SPSS19.0 software (IBM, Armonk, NY, USA). The Shannon-Weaver diversity index $\left(H^{\prime}\right)$ used to characterize the phenotypic frequencies of the characters was defined as: $H^{\prime}=-\sum \mathrm{P}_{\mathrm{i}} \ln \mathrm{P}_{\mathrm{i}}$, where $\mathrm{P}_{\mathrm{i}}$ is the proportion of the individual in the ith class of an n-class character [4].

\section{Results}

\subsection{Variation and diversity of phenotypic traits}

Significant differences were detected among all quantitative parameters for 50 Amomum tsao-ko individual (Table 1). Among thirteen quantitative variables, coefficients of variation $(C V)$ for fruiting rate, fresh fruit weight, dry fruit weight, and dry fruit peel weight and were more than $20 \%$. The greatest variance was obtained in fruiting rate and the $C V$ value was $43.86 \%$. The minimum coefficient of variation was recorded in fresh fruit width and was $7.60 \%$. Shannon-Weaver diversity index $\left(H^{\prime}\right)$ of 13 traits ranged from 1.71 to 2.22 , which was higher in five quantitative traits including seed regiment weight (2.22), dry fruit weight (2.21), dry fruit width (2.15), dry fruit length (2.12), ratio of dry fruit length and width (2.11). These characteristics showed significant phenotypic diversity in Amomum tsao-ko individual.

Table 1 Quantitative traits variation and Shannon-Weaver diversity index $\left(\mathrm{H}^{\prime}\right)$ of Amomum tsao-ko.

\begin{tabular}{|c|c|c|c|c|c|c|c|}
\hline Trait & Min & Max & $\mathrm{R}$ & Mean & SD & $C V / \%$ & $H^{\prime}$ \\
\hline Number of flowers per ear & 48.33 & 121.00 & 72.67 & 82.99 & 15.35 & 18.50 & 2.09 \\
\hline Fruiting rate/\% & 4.33 & 46.97 & 42.64 & 22.40 & 9.82 & 43.86 & 2.09 \\
\hline Fresh fruit weight/g & 6.44 & 21.58 & 15.14 & 10.37 & 3.12 & 30.11 & 1.71 \\
\hline Fresh fruit length/mm & 26.53 & 44.97 & 18.45 & 32.65 & 3.36 & 10.28 & 1.77 \\
\hline Fresh fruit width/mm & 21.47 & 29.50 & 8.03 & 24.34 & 1.85 & 7.60 & 1.87 \\
\hline Ratio of fresh fruit length and width & 1.05 & 1.92 & 0.87 & 1.35 & 0.16 & 12.15 & 1.84 \\
\hline Dry fruit length/mm & 25.10 & 44.88 & 17.78 & 34.75 & 4.28 & 12.32 & 2.12 \\
\hline Dry fruit width/mm & 14.03 & 22.44 & 8.42 & 18.06 & 2.02 & 11.17 & 2.15 \\
\hline Ratio of dry fruit length and width & 1.36 & 2.86 & 1.50 & 1.95 & 0.33 & 16.99 & 2.11 \\
\hline Dry fruit weight/g & 2.00 & 4.80 & 2.80 & 3.31 & 0.74 & 22.37 & 2.21 \\
\hline Dry fruit peel weight/g & 0.54 & 1.58 & 1.04 & 0.92 & 0.24 & 26.40 & 2.07 \\
\hline Seed regiment weight/g & 1.35 & 3.62 & 2.28 & 2.39 & 0.58 & 24.04 & 2.22 \\
\hline Number of seeds per fruit & 23.20 & 51.20 & 28.00 & 37.42 & 6.33 & 16.90 & 2.04 \\
\hline
\end{tabular}

Min, Minimum; Max, Maximum; R, Range; Mean, Average; SD, Standard deviation; CV, Coefficient of variation; $H^{\prime}$, Shannon-Weaver diversity index 


\subsection{Principal component analysis}

Principal components analysis (PCA) effectively explained the variation among different quantitative traits. The first four principal components of data accounted for $78.381 \%$ of the total variance among plants with Eigenvalues $>1$ (Table 2). The first principal component (PC1) explained $34.272 \%$ of the total variance, which was contributed by dry fruit weight, fresh fruit width, dry fruit peel weight and seed regiment weight, these traits are related to yield. PC2 explained $26.293 \%$ of the total variation, with the main contributions from fresh fruit length, dry fruit length and ratio of dry fruit length and width, these are related to the shape of the fruit. PC 3 and PC4 contributed 10.087\% and $7.783 \%$ of total altitude variance, respectively. The number of seeds per fruit was larger contributors to PC3, while the fresh fruit weight were major contributors to variance of the fourth PC.

Table 2 The eigenvalue and contribution rate of principal component based on phenotypic traits of Amomum tsao-ko.

\begin{tabular}{|c|c|c|c|c|}
\hline Traits & PC1 & PC2 & PC3 & PC4 \\
\hline Number of flowers per ear & 0.403 & 0.176 & -0.477 & 0.375 \\
\hline Fruiting rate & -0.389 & 0.459 & 0.424 & 0.024 \\
\hline Fresh fruit weight & 0.247 & 0.035 & 0.388 & 0.84 \\
\hline Fresh fruit length & 0.093 & 0.912 & -0.202 & -0.058 \\
\hline Fresh fruit width & 0.864 & 0.012 & 0.147 & 0.058 \\
\hline Ratio of fresh fruit length and width & -0.439 & 0.777 & -0.278 & -0.077 \\
\hline Dry fruit length & 0.018 & 0.909 & 0.098 & 0.045 \\
\hline Dry fruit width & 0.86 & -0.099 & -0.027 & -0.135 \\
\hline Ratio of dry fruit length and width & -0.526 & 0.738 & 0.097 & 0.126 \\
\hline Dry fruit weight & 0.883 & 0.366 & -0.026 & -0.102 \\
\hline Dry fruit peel weight & 0.835 & 0.161 & 0.056 & -0.013 \\
\hline seed regiment weight & 0.784 & 0.404 & -0.055 & -0.125 \\
\hline Number of seeds per fruit & 0.16 & 0.173 & 0.766 & -0.298 \\
\hline Eigenvalue & 4.455 & 3.411 & 1.311 & 1.012 \\
\hline Contrbution (\%) & 34.272 & 26.293 & 10.087 & 7.783 \\
\hline Accumulated contribution (\%) & 34.272 & 60.511 & 70.598 & 78.381 \\
\hline
\end{tabular}

\section{Discussion}

Amomum tsao-ko has a long history of cultivation in China, especially in southeastern Yunnan Province [5], it is traditional medicinal and condiment. At present, the research on the Amomum tsao-ko is mainly concentrated on chemical composition analysis [6-8]. In present study, Amomum tsao-ko individual showed significant phenotypic diversity in Lvchun County of Yunnan Province (Table 1). Rich phenotypic diversity may be attributed to (1) the species occurs wild in sparse forest from 1,000 to $1,800 \mathrm{~m}$ and is also cultivated in its native range, the habitat is in a wild and semi-wild environment, complex natural environments lead to diversity of phenotypic variation, and/or (2) Amomum tsao-ko is a cross-pollinated crop, natural cross-fertilization as a result of growing mixed genotypes. According to the principal component analysis (PCA) of 50 Amomum tsao-ko germplasm resources, the first four principal components reflected most of the information of 13 traits, and the cumulative contribution rate was $78.381 \%$, each principal component contains trait information has certain relevance. Eigenvalues had significantly different for 13 traits in four principal components, indicating that the PCA results were related to germplasm resources and the choice of traits. The 7 major traits such as fresh fruit length, fresh fruit width, fresh fruit weight, dry fruit length, dry fruit width, dry fruit weight and dry fruit peel weight were the main basis for the actual morphological 
classification. Our study will provide a theoretical basis for the protection and utilization of Yunnan Amomum tsao-ko resources.

\section{Acknowledgments}

This paper is funded by National Natural Science Foundation of China (31460380), Honghe University $\mathrm{PhD}$ and Master Programs (14bs17), Honghe University Youth Backbone of Academic Training Project (2014GG0101). Yunnan College Students Innovation and Entrepreneurship Training Program (201564, DCXM153004), Honghe University Innovative Practice Teaching Reform Pilot Project (CXSJ1304).

\section{References}

[1] Shi Y, Jin H, Yang Y, et al. Herbal Verification of Medicinal Amomum tsao-ko[J]. Medicinal Plant, 2014, 5(9): 16-19, 24.

[2] Lim T K. Amomum tsao-ko[M]//Edible Medicinal And Non-Medicinal Plants. Springer Netherlands, 2013: 813-817.

[3] Wu J, Zhang C, Chen J, et al. Morphological diversity within litchi (Litchi chinensis Sonn.) based on leaf and branch traits[J]. Scientia Horticulturae, 2016, 207: 21-27.

[4] Keylock C J. Simpson diversity and the Shannon-Wiener index as special cases of a generalized entropy [J]. Oikos, 2005, 109(1): 203-207.

[5] Yang Y W, Yang Z Y, Yan M R, et al. Isolation and characterization of microsatellite markers for Amomum tsaoko (Zingiberaceae), an economically important plant in China[J]. Genetics and Molecular Research, 2014, 13(4): 8220-8224.

[6] Liu X L, Qiu H Y, Wang Q, et al. Qualitative study on chemical constituents of Amomum tsao-ko [J]. China Condiment, 2011, 36(1): 104-106.

[7] Li W, Wang P J, Shigematsu M, et al. Chemical composition and antimicrobial activity of essential oil from Amomum tsao-ko cultivated in Yunnan area[C]//Advanced Materials Research. Trans Tech Publications, 2011, 183: 910-914.

[8] Yang Y, Yan R W, Cai X Q, et al. Chemical composition and antimicrobial activity of the essential oil of Amomum tsao-ko[J]. Journal of the Science of Food and Agriculture, 2008, 88(12): 2111-2116. 